\title{
The role of $n-3$ LCPUFA in pregnancy
}

\section{Maria MAKRIDES}

\author{
Women's and Children's Health \\ Research Institute, \\ Women's and Children's Health \\ Network, \\ 72 King William Road, \\ North Adelaide, \\ SA 5006. \\ Australia; School of Paediatrics \& \\ Reproductive Health, \\ University of Adelaide, \\ Adelaide, \\ 5005. Australia \\ $<$ maria.makrides@health.sa.gov.au>
}

\begin{abstract}
The metabolic demand for $n-3$ long chain polyunsaturated fatty acids ( $n-3$ LCPUFA), particularly docosahexaenoic acid (22: $6 n-3, D H A$ ) is increased during pregnancy because of the extra needs of the fetus, expanded maternal cell mass and placenta. However, in Western countries maternal dietary n-3 LCPUFA intake in pregnancy is low and it is not clear whether adaptive metabolic mechanisms, such as increased DHA synthesis from precursor fatty acids, are capable of meeting the increased need in pregnancy. Consequently randomized controlled trials have been important to determine whether additional dietary n-3 LCPUFA in pregnancy modifies pregnancy, maternal and infant health outcomes. Supplementation with at least $1 \mathrm{~g} n-3$ LCPUFA per day results in a modest increase in the duration of gestation that may be most evident at the extremes of gestation. Additionally, n-3 LPUFA supplementation of well nourished pregnant women has little benefit in preventing maternal postnatal depression and is unlikely to result in major benefits to the developmental outcomes of young children. Further work in needed to identify the specific "at risk" groups who are most likely to benefit from supplementation.
\end{abstract}

Key words: DHA, pregnancy, pregnancy outcomes, maternal depression, infant development

(Makrides and Gibson, 2000) and the DHA saved from pregnancy amenorrhea. It has not been possible to quantify the positive and negative sides of the DHA balance equation during pregnancy to ascertain whether there is a "true" increased requirement. However, we do know that the DHA intake of women in industrialized countries in generally low and there is little evidence that women change their dietary habits to enhance their DHA intakes in pregnancy. Mean DHA in Western countries is $70-200 \mathrm{mg} /$ day (Denomme et al., 2005; Innis and Elias, 2003; Meyer et al., 2003; Otto et al., 1997; Stark et al., 2005) but in some cases median intake is lower (30-50 mg/day) highlighting a skewed distribution of intakes (Meyer et al., 2003) so that many women have intakes less than the estimated daily accretion of DHA into the fetus during the last trimester of pregnancy. These observations highlight a potential dietary insufficiency of DHA for both mother and baby.

\section{Dietary insufficiency of $n-3$ LCPUFA in pregnancy and maternal depression}

A pooled analysis of cross country data showed a negative associated between the prevalence of postnatal depression and either seafood consumption or breast milk DHA concentration (Hibbeln, 2002). This led to further investigation of the association between n-3 LCPUFA intake during pregnancy and symptoms of postnatal depression using the data available from the Avon Longitudinal Study of Parents and Children (ALSPAC). This report, with data from approximately 14,000 women, suggests that a negligible intake of seafood at 32 weeks gestation was associated with a doubling in depressive symptoms compared with a high to moderate intake seafood intake supplying at least $320 \mathrm{mg} \mathrm{n}-3$ LCPUFA per day (Hibbeln et al., 2003). These observations together with the association between $\mathrm{n}-3$ fatty acid deficiency and reduced brain serotonin in animal

To cite this article: Makrides M. The role of n-3 LCPUFA in pregnancy. OCL 2011; 18(5): 255-258. doi : 10.1684/ocl.2011.0408 
studies highlight the plausibility of the hypothesis that dietary DHA insufficiency may be associated with symptoms of postnatal depression, and clearly highlight the need for well designed randomised controlled trials to establish a cause and effect relationship between increased dietary n-3 LCPUFA and reduced postnatal depression. Although two of the three available trials indicate that n-3 LCPUFA may ameliorate depressive symptoms in the perinatal period, all three trials have some methodological limitations (small numbers and/or open label design) that cannot exclude bias and random error (Llorente et al., 2003; Freeman et al., 2006a; Freeman et al., 2006b).

With this background we conducted the DOMInO (DHA to Optimise Mother Infant Outcome) trial (Makrides et al., 2010). The DOMInO trial included 2399 women and their children from 5 perinatal centres around Australia. Women with singleton pregnancies were allocated to take $3 \times 0.5 \mathrm{~g}$ capsules per day containing either high-DHA fish oil or a blend of three vegetable oils from midpregnancy until birth. One of the key aims was to assess the effect of DHA supplementation during pregnancy on postnatal depression in women, as indicated by a high score on the Edinburgh Postnatal Depression Scale of at 6 weeks or 6 months post-partum. Although we found a lower percentage of women in the DHA group reporting high levels of depressive symptoms, the DHA and control groups did not significantly differ (9.7\% vs $11.2 \%$, respectively; adjusted relative risk $0.85 ; 95 \%$ confidence interval $(\mathrm{Cl}) 0.70$ to $1.02 ; \mathrm{n}=2399, \mathrm{P}=0.09$ ) (Makrides et al., 2010). This suggests that any effect of supplementation during pregnancy on postnatal depression is either small or negligible.

\section{n-3 LCPUFA during pregnancy and neurodevelopmental outcome of the off-spring}

It is almost impossible to consider dietary intakes of n-3 LCPUFA during pregnancy without some consideration of childhood outcome. Indeed there has been renewed interest regarding the intake of n-3 LCPUFA, fish and seafood during pregnancy and the developmental outcome of children. Data from large cohort studies in the USA and the UK, in which dietary intake was measured in pregnancy and development of the offspring assessed, demonstrate that maternal fish intake during pregnancy was positively associated with developmental and behavioral outcomes. Interestingly the data from both cohorts indicate that there may be a threshold (minimum intake) to achieve the beneficial associations between fish intake in pregnancy and child development. For example in the cohort from the USA the developmental advantage was noted when fish intake in pregnancy was greater than 2 fish meals per week (Oken et al., 2008), while in the cohort from the UK, seafood intake greater than $340 \mathrm{~g}$ per week was associated with improved childhood cognition and behavior (Hibbeln et al., 2007). These data add to the debate regarding the relative risks and benefits of fish and seafood intake during pregnancy especially with regard to neurotoxicity from methyl mercury. Hibbeln et al. modeled their analyses to test the US Federal Government advisories for pregnant women to limit their intake of seafood to $<340 \mathrm{~g}$ per week. Oken et al. specifically assessed maternal mercury levels and found that higher mercury concentrations in maternal red cells were independently adversely associated with developmental outcome. Inclusion of both fish and mercury in their statistical model strengthened the positive association between maternal fish intake and early childhood development as well as strengthening the negative associate between maternal mercury concentration and developmental outcome (Oken et al., 2008). Collectively these data highlight an independence of fish and seafood intake from mercury. Interestingly, only $23 \%$ of the mothers who consumed fish more than twice per week were likely to have the highest concentrations of red cell mercury indicating that either these women ate fish low in mercury or that there were other sources of mercury exposure.

Fish and seafood are good dietary sources of n-3 LCPUFA as well as other nutrients that may impact on developmental outcome such as iodine. It is for this reason that randomized intervention trials involving specific nutrient supplementation are important in establishing the potential specific benefits related to individual nutrients. To date there have been four RCTs involving DHA supple- mentation during pregnancy that have measured cognitive development in childhood (Judge et al., 2007; Tofail et al., 2006; Dunstan et al., 2006; Helland et al., 2001) (one trial has multiple publications). All trials involved supplementation of women from mid-pregnancy to delivery or later with a DHA-rich fish oil. Three trials tested doses of DHA ranging 1.2-2.2 $\mathrm{g} / \mathrm{d}$, whereas one supplied $300 \mathrm{mg}$ DHA/day in muesli bars (Judge et al., 2007). Results from these trials were mixed; no difference in early cognitive development was observed in Fagan Infantest at 6 or 9 months, global development at 10 months (Tofail et al., 2006), language or behaviour at 30 months (Dunstan, et al. 2006), or IQ at 7 years (Helland et al., 2008) between the supplemented and control groups. However, improved problem solving at 9 months (Judge et al., 2007), hand-eye coordination at 30 months (Dunstan et al., 2006) and IQ at 4 years (Helland et al., 2003) were reported with DHA-supplementation in pregnancy. However all of the trials had some methodological limitations - all had relatively small sample sizes and were thus were underpowered for studying global cognitive outcomes in childhood. Furthermore, most trials suffered from significant attrition and/or selective loss from the fish oil supplemented group. Significant attrition or selective loss can both interfere with the integrity of the original randomization and hence increase the likelihood of bias. Although it is not possible to draw any robust conclusions regarding the cause and effect relationship of an increased dietary supply of n-3 LCPUFA during pregnancy and the cognitive development of the off-spring, a number of trials internationally have been specifically designed to answer the elusive question of whether DHA supplementation during pregnancy improves neurocognitive outcomes in childhood.

The first of these recent trials to fully report developmental outcomes in young children has been the DOMInO trial (Makrides et al., 2010). Our DOMInO trial showed that while DHA supplementation did no result in significant benefit to mean cognitive scores at 18 months of age, there were significantly fewer children in the DHA group with low cognitive scores $(<85)$, indicative of mildly delayed development, compared with the control group. This 
finding has caused significant interest because it suggests that DHA supplementation may be efficacious only for young children who may be at risk of developmental delays. The suggestion that some children may need more DHA than others is perhaps best highlighted by the special case of the preterm infant (Makrides et al., 2009).

\section{n-3 LCPUFA supplementation during pregnancy, pregnancy duration and birth size}

One of the potential effects of $n-3$ LCPUFA supplementation during pregnancy, which has not received much recent attention, is the prevention of preterm birth or the prolongation of gestation. It has been postulated that n3 LCPUFA could delay initiation of labour and cervical ripening by inhibiting the production of prostaglandins F2 $\alpha$ and E2. This biochemical plausibility taken together with the observational studies showing an association between high fish consumption and increased duration of pregnancy, higher birth weight and a lower incidence of pre-eclampsia (Olsen et al., 1986; Olsen and Joensen, 1985) resulted in a number of randomised controlled trials to assess the cause and effect relationship between fish oil supplementation in pregnancy and improving major pregnancy outcomes.

Three systematic reviews have recently aggregated the results of the relevant randomized controlled trials (Makrides et al., 2006; Horvath et al., 2007; Szajewska et al., 2006). The metaanalyses showed remarkably consistent results despite the fact that these reviews had differing inclusion criteria. In brief, supplementation with marine oil in the second half of pregnancy resulted in a modest increase in the length of gestation (approximately 2.5 days) compared with no marine oil treatment. This was not reflected in a clear difference between the two groups in the risk of preterm birth ( $<37$ weeks gestation), although women allocated marine oil did have a lower risk of giving birth before 34 weeks gestation (Makrides et al., 2006; Horvath et al., 2007). These data are also surprisingly consistent with the data from the DOMInO trial, which is the largest published trial in the field (Makrides et al., 2010). The level of concordance between the outcomes of the systematic reviews and the single largest trial offers a high degree of confidence that relatively high dose dietary n-3 LCPUFA during pregnancy could be an effective strategy to prevent early preterm birth. Of course preventing early preterm birth implies a prolongation of gestation and it is important to consider whether there is an effect at the other extreme of gestation length and whether n-3 LCPUFA supplementation increases post-term birth. Although these data were difficult to decipher from the systematic reviews, the DOMInO trial did suggest that supplementation was associated with increased obstetric intervention (inductions and elective caesarean sections) because of postterm dates (Makrides et al., 2010). Clearly more work is needed and care is required in balancing the potential hazard and benefit of $n-3$ LCPUFA supplementation with regard to the duration of gestation.

Interestingly both the systematic reviews and the DOMInO trial show modest increases in birth weight of infants born to women treated with $n-3$ LCPUFA during pregnancy compared with control (Makrides et al., 2006). These differences were commensurate with the small increases in mean gestation length and there were no overall differences between the groups in the proportion of small-for-gestational age babies or birth weight zscores suggesting that any effect of $n-3$ LCPUFA may be a function of the duration of gestation rather than a direct effect on fetal growth.

In summary, n-3 LCPUFA of pregnant women is well studied and important core information exists. Supplementation, at higher n-3 LCPUFA doses than available in commercially available prenatal supplements, results in a modest increase in the duration of gestation that may be most evident at the extremes of gestation. Additionally, n-3 LPUFA supplementation of well nourished pregnant women is unlikely to have a major effect of preventing maternal postnatal depression and is unlikely to result in major benefits to the developmental outcomes of young children. Further work in needed to identify the specific "at risk" groups who are most likely to benefit from supplementation.

\section{REFERENCES}

Burdge GC, Calder PC. Conversion of alphalinolenic acid to longer-chain polyunsaturated fatty acids in human adults. Reprod Nutr Dev 2005; 45: 581-97.

Denomme J, Stark KD, Holub BJ. Directly quantitated dietary (n-3) fatty acid intakes of pregnant Canadian women are lower than current dietary recommendations. J Nutr 2005; 135: 206-11.

Dunstan JA, Simmer K, Dixon G, Prescott SL. Cognitive assessment at $21 / 2$ years following fish oil supplementation in pregnancy: a randomized controlled trial. Arch Dis Fetal \& Neonatal Ed 2006; 93: F45-50.

Freeman MP, Hibbeln JR, Wisner KL, Brumbach BH, Watchman M, Gelenberg AJ. Randomized dose-ranging pilot trial of omega-3 fatty acids for postpartum depression. Acta Psychiatr Scand 2006; 113: 31-5.

Freeman MP, Hibbeln JR, Wisner KL, Watchman M, Gelenberg AJ. An Open Trial of omega-3 fatty acids for depression in pregnancy. Acta Neuropsychiatrica 2006; 18: 21-4.

Helland IB, Saugstad OD, Smith L, et al. Similar effects on infants of n-3 and n- 6 fatty acids supplementation to pregnant and lactating women. Pediatrics 2001; 108: 1-10.

Helland IB, Smith L, Saarem K, Saugstad OD, Drevon CA. Maternal supplemenatation with very-long-chain $\mathrm{n}-3$ fatty acids during pregnancy and lactation augments children's IQ at 4 years of age. Pediatrics 2003; 111: e39-44.

Helland IB, Smith L, Blomen B, Saarem K, Saugstad OD, Drevon CA. Effect of supplementing pregnant and lactating mothers with $n-3$ very-long-chain fatty acids on children's IQ and body mass index at 7 years of age. Pediatrics 2008; 122: e472-9.

Hibbeln JR. Seafood consumption, the DHA content of mothers' milk and prevalence rates of postpartum depression: a cross-national, ecological: a cross-national, ecological analysis. J Affect Disord 2002; 69: 15-29.

Hibbeln JR, Davis JM, Heron J, Evans J, Wolke DFH, Golding J, ALSPAC study team. Low dietary omega-3s and increased depression risk in 14,541 pregnancies. Abstract [NR418] - American Psychiatric Association 2003 Annual Meeting.

Hibbeln JR, Davis JM, Steer C, et al. Maternal seafood consumption in pregnancy and neurodevelopmental outcomes in childhood (ALSPAC study): an observational cohort study. Lancet 2007; 369: 578-85.

Horvath A, Koletzko B, Szajewska H. Effect of supplementation of women in high-risk pregnancies with long-chain polyunsaturated 
fatty acids on pregnancy outcomes and growth measures at birth: a meta-analysis of randomized controlled trials. Br J Nutr 2007; 98: 253-9.

Innis SM. Perinatal biochemistry and physiology of long-chain polyunsaturated fatty acids. J Pediatr 2003; 143: S1-8.

Innis SM, Elias SL. Intakes of essential n-6 and n-3 polyunsaturated fatty acids among pregnant Canadian women. Am J Clin Nutr 2003; 77: 473-8.

Judge MP, Harel O, Lammi-Keefe CJ. Maternal consumption of a docosahexaenoic acidcontaining functional food during pregnancy: benefit for infant performance on problem-solving but not on recognition memory tasks at age 9 mo. Am J Clin Nutr 2007; 85: 1572-7.

Llorente AM, Jensen CL, Voigt RG, Fraley JK, Berretta MC, Heird WC. Effect of maternal docosahexaenoic acid supplementation on postpartum depression and information processing. Am J Obstet Gynecol 2003; 188: 1348-53.

Makrides M, Gibson RA. Long-chain polyunsaturated fatty acid requirements during pregnancy and lactation. Am J Clin Nutr 2000; 71: 307-11.
Makrides M, Gibson RA, McPhee AJ, et al. Neurodevelopmental outcomes of preterm infants fed high-dose docosahexaenoic acid: a randomized controlled trial. JAMA 2009; 301: 175-82.

Meyer BJ, Mann NJ, Lewis JL, Milligan GC, Sinclair AJ, Howe PR. Dietary intakes and food sources of omega- 6 and omega- 3 polyunsaturated fatty acids. Lipids 2003; 38: 391-8.

Makrides M, Duley L, Olsen SF. Marine oil, and other prostaglandin precursor, supplementation for pregnancy uncomplicated by pre-eclampsia or intrauterine growth restriction. Cochrane Database Syst Rev 2006; 3: CD003402.

Makrides M, Gibson RA, McPhee AJ, Yelland L, Quinlivan J, Ryan P. Effect of DHA supplementation during pregnancy on maternal depression and neurodevelopment of young children: a randomized controlled trial. JAMA 2010; 304: 1675-83.

Oken E, Radesky JS, Wright RO, et al. Maternal fish intake during pregnancy, blood mercury levels, and child cognition at age 3 years in a US cohort. Am / Epidemiol 2008; 167: 1171-81.

Olsen SF, Hansen HS, Sorensen TIA, et al. Intake of marine fat, rich in (n-3)-polyunsa- turated fatty acids, may increase birthweight by prolonging gestation. Lancet 1986; 2: 367-9.

Olsen SF, Joensen HD. High liveborn birth weights in the Faroes: a comparison between birth weights in the Faroes and in Denmark. Epidemiol Community Health 1985; 39: 27-32.

Otto SJ, Houwelingen AC, Antal M, et al. Maternal and neonatal essential fatty acid status in phospholipids: an international comparative study. Eur J Clin Nutr 1997; 51: 232-42.

Stark KD, Beblo S, Murthy M, et al. Comparison of bloodstream fatty acid composition from African-American women at gestation, delivery, and postpartum. / Lipid Res 2005; 46: 516-25.

Szajewska H, Horvath A, Koletzko B. Effect of n-3 long-chain polyunsaturated fatty acid supplementation of women with low-risk pregnancies on pregnancy outcomes and growth measures at birth: a meta-analysis of randomized controlled trials. Am / Clin Nutr 2006; 83: 1337-44.

Tofail F, Kabir I, Hamadani JD, et al. Supplementation of fish-oil and soy-oil during pregnancy and psychomotor development of infants. J Health Popul Nutr 2006; 24: 48-56. 